\title{
ON THE REFLECTED RANDOM WALK ON $\mathbb{R}_{+}$
}

\author{
JEAN-BAPTISTE BOYER ${ }^{1}$
}

\begin{abstract}
Let $\rho$ be a borelian probability measure on $\mathbb{R}$ having a moment of order 1 and a drift $\lambda=\int_{\mathbb{R}} y \mathrm{~d} \rho(y)<0$. Consider the random walk on $\mathbb{R}_{+}$starting at $x \in \mathbb{R}_{+}$and defined for any $n \in \mathbb{N}$ by

$$
\left\{\begin{aligned}
X_{0} & =x \\
X_{n+1} & =\left|X_{n}+Y_{n+1}\right|
\end{aligned}\right.
$$

where $\left(Y_{n}\right)$ is an iid sequence of law $\rho$. We denote $P$ the Markov operator associated to this random walk and, for any borelian bounded function $f$ on $\mathbb{R}_{+}$, we call Poisson's equation the equation $f=g-P g$ with unknown function $g$. In this paper, we prove that under a regularity condition on $\rho$ and $f$, there is a solution to Poisson's equation converging to 0 at infinity. Then, we use this result to prove the functional central limit theorem and it's almost-sure version.
\end{abstract}

Mathematics Subject Classification. 60J10.

Received April 29, 2016. Revised November 24, 2016. Accepted May 12, 2017.

\section{INTRODUCTION}

Let $\rho$ be a borelian probability measure on $\mathbb{R}$ having a moment of order 1 and a negative drift $\lambda=$ $\int_{\mathbb{R}} y \mathrm{~d} \rho(y)<0$.

Consider the random walk on $\mathbb{R}_{+}$starting at $x \in \mathbb{R}_{+}$and defined for any $n \in \mathbb{N}$ by

$$
\left\{\begin{aligned}
X_{0} & =x \\
X_{n+1} & =\left|X_{n}+Y_{n+1}\right|
\end{aligned}\right.
$$

where $\left(Y_{n}\right)$ is an iid sequence of law $\rho$.

We denote $P$ the Markov operator associated to this random walk. This is the operator defined for any borelian and bounded function $f$ on $\mathbb{R}_{+}$and any $x \in \mathbb{R}_{+}$by

$$
P f(x)=\int_{\mathbb{R}} f(|x+y|) \mathrm{d} \rho(y) .
$$

The aim of this article is to study this Markov chain and to do so, we will use a standard technique (known as Gordin's method) which consists in finding a solution $g$ to the "Poisson equation" $g-P g=f$ for $f$ in a certain Banach space.

Keywords and phrases. Markov chains, Poisson's equation, Gordin's method, renewal theorem, random walk on the half line.

1 IMB, Université de Bordeaux / MODAL'X, Université Paris-Ouest, Nanterre, France. jeaboyer@math.cnrs.fr 
Using results of Glynn and Meyn, we will prove that under a regularity assumption on the measure $\rho$, there always is a solution to this equation if $f$ is borelian and bounded but this solution will not be bounded in general and this prevents us from studying the large deviation principle and complicates the study of the central limit theorem.

However, we will see in Section 2 that the solution to Poisson's equation satisfies some equations (see Prop. 2.3) and using a "stopped" renewal theorem that we will state in Section 3 (see Cor. 3.6) we will prove the following

Corollary 1.1 (4.7). Let $\rho$ be an absolutely continuous probability measure on $\mathbb{R}$ having a moment of order 1 , a negative drift $\lambda=\int_{\mathbb{R}} y \mathrm{~d} \rho(y)<0$ and such that $\rho\left(\mathbb{R}_{+}^{*}\right)>0$. Let $\left(X_{n}\right)$ be the reflected random walk on $\mathbb{R}_{+}$ defined by $\rho$.

Let $\nu$ be the unique stationary probability measure on $\mathbb{R}_{+}$(it exists according to [6] or [8]).

Then, for any directly Riemann-integrable function $f$ on $\mathbb{R}_{+}$such that $\int f \mathrm{~d} \nu=0$, there is a bounded and a.e. continuous function $g$ on $\mathbb{R}_{+}$such that

$$
f=g-P g \text { and } \lim _{x \rightarrow+\infty} g(x)=0
$$

This will allow us to prove the following

Theorem 1.2 (4.8 and 4.11). Let $\rho$ be an absolutely continuous probability measure on $\mathbb{R}$ having a moment of order 1 , a negative drift $\lambda=\int_{\mathbb{R}} y \mathrm{~d} \rho(y)<0$ and such that $\rho\left(\mathbb{R}_{+}^{*}\right)>0$.

Let $\left(X_{n}\right)$ be the reflected random walk on $\mathbb{R}_{+}$defined by $\rho$.

For any directly Riemann-integrable function $f$ on $\mathbb{R}_{+}$such that $\int f \mathrm{~d} \nu=0$, there are constants $C_{1}, C_{2} \in \mathbb{R}_{+}^{*}$ such that for any $\varepsilon \in] 0,1]$, any $x \in \mathbb{R}_{+}$and any $n \in \mathbb{N}^{*}$,

$$
\mathbb{P}_{x}\left(\left\{\left|\frac{1}{n} \sum_{k=0}^{n-1} f\left(X_{k}\right)\right| \geqslant \varepsilon\right\}\right) \leqslant C_{1} \mathrm{e}^{-C_{2} \varepsilon^{2} n}
$$

In particular, for any $x \in \mathbb{R}_{+}$,

$$
\frac{1}{n} \sum_{k=0}^{n-1} f\left(X_{k}\right) \rightarrow 0 \quad \mathbb{P}_{x} \text {-a.e. and in } \mathrm{L}^{1}\left(\mathbb{P}_{x}\right)
$$

Moreover, if $\rho$ as a moment of order $2+\varepsilon$ for some $\varepsilon \in \mathbb{R}_{+}^{*}$, then, for any directly Riemann-integrable function $f$ on $\mathbb{R}_{+}$with $\int f \mathrm{~d} \nu=0$ we denote $g$ the bounded function given by Corollary 4.7 and such that $f=g-P g$ and we set

$$
\sigma^{2}=\int_{\mathbb{R}_{+}} g^{2}-(P g)^{2} \mathrm{~d} \nu
$$

and for any $x \in \mathbb{R}_{+}$and any $n \in \mathbb{N}^{*}$, we set $S_{n} f(x)=\sum_{k=0}^{n-1} f\left(X_{k}\right)$ where $\left(X_{n}\right)$ is the reflected random walk defined by $\rho$ and starting at $x$.

Then, if $\sigma^{2}=0$, we have that for a.e. any $x \in \mathbb{R}_{+},\left(S_{n} f(x)\right)$ is bounded in $\mathrm{L}^{\infty}\left(\mathbb{P}_{x}\right)$.

On the other hand, if $\sigma^{2} \neq 0$, we set, for $t \in[0,1]$ and any $x \in \mathbb{R}_{+}$,

$$
\xi_{n}(t)=\frac{1}{\sqrt{n}}\left(S_{i} f(x)+n\left(t-\frac{i}{n}\right) f\left(X_{i}\right)\right) \quad \text { for } \quad \frac{i}{n} \leqslant t \leqslant \frac{i+1}{n} \quad \text { and } \quad 0 \leqslant i \leqslant n-1
$$

and we have that, for any bounded continuous function $F: \mathcal{C}^{0}([0,1]) \rightarrow \mathbb{R}$,

$$
\mathbb{E} F\left(\xi_{n}\right) \rightarrow \mathbb{E} F\left(W_{\sigma^{2}}\right) \text { and } \frac{1}{\ln n} \sum_{k=1}^{n} \frac{1}{k} F\left(\xi_{k}\right) \rightarrow \mathbb{E} F\left(W_{\sigma^{2}}\right) \text { a.e. }
$$

Where we denoted $W_{\sigma^{2}}$ the Wiener measure with variance $\sigma^{2}$. 
Moreover, for any a.e. continuous function $\varphi$ on $\mathbb{R}$ such that $x^{2} \varphi(x)$ is bounded,

$$
\frac{1}{\ln n} \sum_{k=1}^{n} \frac{1}{k} \varphi\left(\frac{S_{k} f(x)}{\sqrt{k}}\right) \rightarrow \mathbb{E} \varphi\left(W_{\sigma^{2}}(1)\right) \text { a.e. }
$$

Remark 1.3. The functional central limit theorem and the almost sure functional central limit theorem that we state here have many corollaries such as the CLT (taking $F_{\varphi}(\xi)=\varphi(\xi(1)$ ) for any continuous and bounded function $\varphi$ on $\mathbb{R}$ ), the law of the iterated logarithm (see Thm. 2.4 in [1]), a control of $\frac{\max _{k \in[0, n]} S_{k} f(x)}{\sqrt{n}}$ (taking $\left.F(\xi):=\sup _{t \in[0,1]} \xi(t)\right)$, or an estimation of $\sigma^{2}$ (taking $\left.\varphi(x)=x^{2}\right)$.

\section{InduCED MaRKov Chains}

The aim of this section is to study the process of induction of Markov chains by stopping times and to link the induced chain to the original one. We study it in a general case as it doesn't use any particular property of the reflected random walk.

\subsection{Definitions}

Let $\left(X_{n}\right)$ be a Markov chain on a standard Borel space $\mathbf{X}$. We define a Markov operator on $\mathbf{X}$ setting, for a borelian function $f$ and $x \in \mathbf{X}$,

$$
P f(x)=\mathbb{E}\left[f\left(X_{1}\right) \mid X_{0}=x\right]
$$

Given a stopping time $\tau$, we can study the Markov chain $\left(X_{\tau^{n}}\right)_{n \in \mathbb{N}}$ where $\tau^{n}$ is defined by

$$
\left\{\begin{aligned}
\tau^{0}\left(\left(X_{n}\right)\right) & =0 \\
\tau^{k+1}\left(\left(X_{n}\right)\right) & =\tau^{k}\left(\left(X_{n}\right)\right)+\tau\left(\theta^{\tau^{k}\left(\left(X_{n}\right)\right)}\left(X_{n}\right)\right)
\end{aligned}\right.
$$

where $\theta$ stands for the shift on $\mathbf{X}^{\mathbb{N}}$.

We denote $Q$ the sub-Markov operator associated to $\left(X_{\tau^{n}}\right)$, that is, for a borelian function $g$ on $\mathbf{X}$ and $x \in \mathbf{X}$,

$$
Q(g)(x)=\int_{\{\tau<+\infty\}} g\left(X_{\tau}\right) \mathrm{d} \mathbb{P}_{x}\left(\left(X_{n}\right)\right) .
$$

If, for any $x \in \mathbf{X}, \mathbb{P}_{x}(\{\tau<+\infty\})=1$, then $Q$ is a Markov operator.

Finally, we define two other operators on $\mathbf{X}$ setting, for a borelian non negative function $f$ and $x \in \mathbf{X}$,

$$
\begin{array}{r}
S f(x)=\int_{\{\tau=1\}} f\left(X_{1}\right) \mathrm{d} \mathbb{P}_{x}\left(\left(X_{n}\right)\right) \\
R f(x)=\int_{\{\tau<+\infty\}} f\left(X_{0}\right)+\ldots+f\left(X_{\tau-1}\right) \mathrm{d} \mathbb{P}_{x}\left(\left(X_{n}\right)\right)
\end{array}
$$

Definition 2.1 ( $\theta$-compatible stopping times).

We say that a stopping time $\tau$ is $\theta$-compatible if for all $x \in \mathbf{X}, \mathbb{P}_{x}(\{\tau=0\})=0$ and for $\mathbb{P}_{x}-$ a.e. $\left(X_{n}\right) \in \mathbf{X}^{\mathbb{N}}$, $\tau\left(\left(X_{n}\right)\right) \geqslant 2$ implies that $\tau\left(\theta\left(X_{n}\right)\right)=\tau\left(\left(X_{n}\right)\right)-1$.

Example 2.2. Let $\mathbf{Y}$ be a borelian subset of $\mathbf{X}$ and $\tau_{\mathbf{Y}}$ the time of first return in $\mathbf{Y}$ :

$$
\tau_{\mathbf{Y}}\left(\left(X_{n}\right)\right)=\inf \left\{n \in \mathbb{N}^{*} ; X_{n} \in \mathbf{Y}\right\}
$$

Then, $\tau_{\mathbf{Y}}$ is $\theta$-compatible.

Moreover, $\tau_{\mathbf{Y}}^{n}$ as we defined it coresponds to the time of $n$-th return to $\mathbf{Y}$. 
For $x \in \mathbf{X}$, we set $u(x)=\mathbb{E}_{x} \tau_{\mathbf{Y}}$ and we call $\mathbf{Y}$ strongly Harris-recurrent if $u$ is finite on $\mathbf{X}$. This imply in particular that for any $x$ in $\mathbf{X}, \tau_{\mathbf{Y}}$ is $\mathbb{P}_{x}-$ a.e. finite.

Indeed for any borelian non negative function $f$ and any $x \in \mathbf{X}$, we have that

$$
\begin{aligned}
Q f(x)= & \int_{\{\tau<+\infty\}} f\left(X_{\tau}\right) \mathrm{d} \mathbb{P}_{x}=\sum_{n=1}^{+\infty} \mathbb{E}_{x} f\left(X_{n}\right) \mathbf{1}_{\{\tau=n\}}=\sum_{n=1}^{+\infty} \mathbb{E}_{x} f\left(X_{n}\right) \mathbf{1}_{\mathbf{Y}^{c}}\left(X_{1}\right) \ldots \mathbf{1}_{\mathbf{Y}^{c}}\left(X_{n-1}\right) \mathbf{1}_{\mathbf{Y}}\left(X_{n}\right) \\
= & \sum_{n=1}^{+\infty}\left(P \mathbf{1}_{\mathbf{Y}^{c}}\right)^{n-1} P\left(f \mathbf{1}_{\mathbf{Y}}\right)=\sum_{n=0}^{+\infty}\left(P \mathbf{1}_{\mathbf{Y}^{c}}\right)^{n} P\left(f \mathbf{1}_{\mathbf{Y}}\right) \\
R f(x)= & \int_{\{\tau<+\infty\}} f\left(X_{0}\right)+\ldots+f\left(X_{\tau-1}\right) \mathrm{d} \mathbb{P}_{x}=\sum_{n=0}^{+\infty} \mathbb{E}_{x} f\left(X_{n}\right) \mathbf{1}_{\{\tau \geqslant n+1\}} \\
= & f(x)+\sum_{n=1}^{+\infty} \mathbb{E}_{x} f\left(X_{n}\right) \mathbf{1}_{\mathbf{Y}^{c}}\left(X_{1}\right) \ldots \mathbf{1}_{\mathbf{Y}^{c}}\left(X_{n}\right)=f(x)+\sum_{n=1}^{+\infty}\left(P \mathbf{1}_{\mathbf{Y}^{c}}\right)^{n} f(x)=\sum_{n=0}^{+\infty}\left(P \mathbf{1}_{\mathbf{Y}^{c}}\right)^{n} f(x) \\
& S f(x)=\int_{\{\tau=1\}} f\left(X_{1}\right) \mathrm{d} \mathbb{P}_{x}=\int f\left(X_{1}\right) \mathbf{1}_{\mathbf{Y}}\left(X_{1}\right) \mathrm{d} \mathbb{P}_{x}=P\left(f \mathbf{1}_{\mathbf{Y}}\right)
\end{aligned}
$$

Thus, we have that $(R+Q) f=\left(I_{d}+R P\right) f, R S f=Q f,(P-S) Q f=P\left(\mathbf{1}_{\mathbf{Y}^{c}} Q f\right)=Q f-S f$ and $(P-S) R f=$ $P\left(\mathbf{1}_{\mathbf{Y}^{c}} R f\right)=R f-f$.

Note that $P, Q, R, S, P-S, Q-S$ and $R-I_{d}$ are positive operators and so the computations we made make sense for any non negative borelian function $f$.

Next lemma generalizes those relations for any $\theta$-compatible stopping time.

Proposition 2.3. Let $\tau$ be a $\theta$-compatible stopping time such that for any $x \in \mathbf{X}, \tau$ is $\mathbb{P}_{x}-$ a.e. finite.

For any non negative borelian function $f$ on $\mathbf{X}$, we have:

$$
\begin{aligned}
(R+Q) f & =\left(I_{d}+R P\right) f \\
\left(I_{d}+P R\right) f & =\left(I_{d}+S\right) R f \\
\left(I_{d}+S\right) Q f & =(S+P Q) f \\
R S f & =Q f
\end{aligned}
$$

Proof. Let $f$ be a borelian non negative function on $\mathbf{X}$ and $x \in \mathbf{X}$.

Using the Markov property and $\tau$ being a $\theta$-compatible stopping time, we have that for any $n \in \mathbb{N}^{*}$,

$$
\mathbb{E}_{x} f\left(X_{n}\right) \mathbf{1}_{\{\tau \geqslant n\}}=\mathbb{E}_{x} \operatorname{Pf}\left(X_{n-1}\right) \mathbf{1}_{\{\tau \geqslant n\}}
$$

And so,

$$
\begin{aligned}
(R+Q) f(x) & =\mathbb{E}_{x} f\left(X_{0}\right)+\ldots+f\left(X_{\tau}\right) \mathrm{d} \mathbb{P}_{x}=\mathbb{E}_{x} \sum_{n=0}^{+\infty} f\left(X_{n}\right) \mathbf{1}_{\{\tau \geqslant n\}} \\
& =f(x)+\sum_{n=1}^{+\infty} \mathbb{E}_{x} \operatorname{Pf}\left(X_{n-1}\right) \mathbf{1}_{\{\tau \geqslant n\}}=f(x)+\operatorname{RPf}(x)
\end{aligned}
$$


Moreover, as $\tau$ is $\theta$-compatible,

$$
\int_{\{\tau \geqslant 2\}} R f\left(X_{1}\right) \mathrm{d} \mathbb{P}_{x}\left(\left(X_{n}\right)\right)=\int_{\{\tau \geqslant 2\}} f\left(X_{1}\right)+\ldots+f\left(X_{\tau-1}\right) \mathrm{d} \mathbb{P}_{x}\left(\left(X_{n}\right)\right) .
$$

Thus,

$$
\begin{aligned}
f(x)+P R f(x) & =f(x)+\int_{\{\tau=1\}} R f\left(X_{1}\right) \mathrm{d} \mathbb{P}_{x}\left(\left(X_{n}\right)\right)+\int_{\{\tau \geqslant 2\}} R f\left(X_{1}\right) \mathrm{dP}_{x}\left(\left(X_{n}\right)\right) \\
& =f(x)+S R f(x)+\int_{\{\tau \geqslant 2\}} f\left(X_{1}\right)+\ldots+f\left(X_{\tau-1}\right) \mathrm{d} \mathbb{P}_{x}\left(\left(X_{n}\right)\right) \\
& =S R f(x)+\int f\left(X_{0}\right)+\ldots+f\left(X_{\tau-1}\right) \mathrm{d} \mathbb{P}_{x}\left(\left(X_{n}\right)\right)=S R f(x)+R f(x) .
\end{aligned}
$$

Then, by definition of $S, \int_{\{\tau=1\}} f\left(X_{1}\right) \mathrm{d} \mathbb{P}_{x}\left(\left(X_{n}\right)\right)=\int_{\{\tau=1\}} f\left(X_{\tau}\right) \mathrm{d} \mathbb{P}_{x}\left(\left(X_{n}\right)\right)$, so,

$$
S f(x)+P Q f(x)=\int \mathbf{1}_{\{\tau=1\}}\left(f\left(X_{1}\right)+Q f\left(X_{1}\right)\right)+\mathbf{1}_{\{\tau \geqslant 2\}} f\left(X_{\tau}\right) \mathrm{d} \mathbb{P}_{x}\left(\left(X_{n}\right)\right)=S Q f(x)+Q f(x) .
$$

Finally, using Markov's property, we have that for any $n \in \mathbb{N}^{*}$,

$$
\mathbb{E}_{x} S f\left(X_{n-1}\right) \mathbf{1}_{\{\tau \geqslant n\}}=\int_{\{\tau=n+1\}} f\left(X_{n+1}\right)
$$

therefore,

$$
\begin{aligned}
R S f(x) & =\mathbb{E}_{x} \sum_{n=1}^{+\infty} S f\left(X_{n-1}\right) \mathbf{1}_{\{\tau \geqslant n\}} \\
& =\sum_{n=1}^{+\infty} \mathbb{E}_{x} S f\left(X_{n-1}\right) \mathbf{1}_{\{\tau \geqslant n\}}=\sum_{n=1}^{\infty} \mathbb{E}_{x} f\left(X_{n+1}\right) \mathbf{1}_{\{\tau=n+1\}}=Q f(x) .
\end{aligned}
$$

And this finishes the proof of this proposition.

Lemma 2.4. Let $\left(X_{n}\right)$ be a Markov chain on a standard borelian space $\mathbf{X}$.

Let $\nu$ be a finite $P$-invariant measure on $\mathbf{X}$ and $\tau$ a $\theta$-compatible stopping time such that for $\nu$-a.e $x \in \mathbf{X}$, $\lim _{n \rightarrow+\infty} \mathbb{P}_{x}(\tau \geqslant n)=0$.

Then, for any non negative borelian function $f$ on $\mathbf{X}$, we have

$$
\int_{\mathbf{X}} f \mathrm{~d} \nu=\int_{\mathbf{X}} S R f \mathrm{~d} \nu
$$

Proof. According to Proposition 2.3, $f+P R f=R f+S R f$. So, if $R f \in \mathrm{L}^{1}(\mathbf{X}, \nu)$, as $\nu$ is $P$-invariant, we get the lemma.

If $f \notin \mathrm{L}^{1}(\mathbf{X}, \nu)$, we will get the lemma by approximation.

First, we assume that $f$ is bounded. In general, $R f \notin \mathrm{L}^{1}(\mathbf{X}, \nu)$ so, we approximate it with a sequence of integrable functions.

More precisely, for $n \in \mathbb{N}^{*}$, we denote by $R_{n}$ the operator defined like $R$ but associated to the stopping time $\min (n, \tau)$ (which is not $\theta$-compatible).

That is to say, for a borelian non negative function $f$ and any $x \in \mathbf{X}$,

$$
R_{n} f(x)=\mathbb{E}_{x} \sum_{k=0}^{\min (\tau, n)-1} f\left(X_{k}\right) .
$$


As $\{\min (\tau, n)=1\}=\{\tau=1\}$ for $n \geqslant 2$, the operator $S$ associated to $\min (\tau, n)$ does not depend on $n$ for $n \geqslant 2$.

As $\min (\tau, n)$ is not $\theta$-compatible, we can't use Proposition 2.3, but we have for $n \geqslant 2$, that

$$
\begin{aligned}
P R_{n} f(x) & =\mathbb{E}_{x} \sum_{k=0}^{\min (\tau \circ \theta, n)-1} f\left(X_{k+1}\right)=S R_{n} f(x)+\int_{\{\tau \geqslant 2\}} \sum_{k=0}^{\min (\tau-1, n)-1} f\left(X_{k+1}\right) \mathrm{d} \mathbb{P}_{x} \\
& =S R_{n} f(x)+\int_{\{\tau \geqslant 2\}} \sum_{k=1}^{\min (\tau, n+1)-1} f\left(X_{k}\right) \mathrm{d} \mathbb{P}_{x}=S R_{n} f+R_{n+1} f-f
\end{aligned}
$$

And, as $f$ is bounded, for any $x \in \mathbf{X},\left|R_{n} f(x)\right| \leqslant n\|f\|_{\infty}$ and so $R_{n} f$ is integrable since $\mu$ is a finite measure and,

$$
\begin{aligned}
\int S R_{n} f-f \mathrm{~d} \nu & =\int P R_{n} f-R_{n+1} f \mathrm{~d} \nu=\int R_{n} f-R_{n+1} f \mathrm{~d} \nu=\int f\left(X_{n}\right) \mathbf{1}_{\{\tau \geqslant n\}} \mathrm{d} \mathbb{P}_{x}\left(\left(X_{n}\right)\right) \mathrm{d} \nu(x) \\
& =\int \operatorname{Pf}\left(X_{n-1}\right) \mathbf{1}_{\{\tau \geqslant n\}} \mathrm{d} \mathbb{P}_{x}\left(\left(X_{n}\right)\right) \mathrm{d} \nu(x)
\end{aligned}
$$

So,

$$
\left|\int S R_{n} f-f \mathrm{~d} \nu\right| \leqslant\|f\|_{\infty} \int_{X} \mathbb{P}_{x}(\{\tau \geqslant n\}) \mathrm{d} \nu(x) \rightarrow 0 \text { (by monotone convergence) }
$$

and using the monotone convergence theorem, we get the expected result for borelian bounded functions.

If $f$ is not bounded and non-negative, we take an increasing sequence $\left(f_{n}\right)$ of bounded positive functions which converges to $f$ and we get the expected result by monotone convergence.

Example 2.5. If $\tau$ is the return time to some strongly Harris-recurrent set $\mathbf{Y}$, then $S f(x)=P\left(f \mathbf{1}_{\mathbf{Y}}\right)(x)$. Moreover for every $P$-invariant measure $\nu$ and every $f \in \mathrm{L}^{1}(\mathbf{X}, \nu)$, such that $R f$ is $\nu$-a.e. finite, $\int_{\mathbf{X}} S R f \mathrm{~d} \nu=$ $\int_{\mathbf{Y}} R f \mathrm{~d} \nu$.

In particular, with $f=1$, we have that, $\int_{\mathbf{Y}} \mathbb{E} \tau \mathrm{d} \nu=\nu(\mathbf{X})$. This is Kac's lemma for dynamical systems.

\subsection{Application to the study of invariant measures}

In this subsection, $\mathbf{X}$ is a complete separable metric space endowed with its Borel $\sigma$-algebra and "measure" stands for "borelian measure". We assume that there exist (at least) a $P$-invariant probability measure on $\mathbf{X}$.

We also fix a $\theta$-compatible stopping time $\tau$ such that for any $x$ in $\mathbf{X}, \mathbb{E}_{x} \tau$ is finite.

For a non-negative operator $T$ defined on borelian functions on $\mathbf{X}$ and a borelian probability measure $\nu$ on $\mathbf{X}$ we denote $T^{*} \nu$ the borelian measure defined by $T^{*} \nu(A)=\int T \mathbf{1}_{A} \mathrm{~d} \nu$.

Lemma 2.6. Let $\mu$ be a finite non-zero $P$-invariant borelian measure on $\mathbf{X}$. Then, $S^{*} \mu$ is a finite non-zero $Q$-invariant measure on $\mathbf{X}$.

Moreover, $R^{*} S^{*} \mu=\mu$ and $S^{*} \mu$ is absolutely continuous with respect to $\mu$.

Proof. First, for any non negative $f \in \mathcal{B}(\mathbf{X})$ and any $x \in \mathbf{X}, S f(x) \leqslant P f(x)$.

So, $\int S f \mathrm{~d} \mu \leqslant \int P f \mathrm{~d} \mu=\int f \mathrm{~d} \mu$ since $\mu$ is $P$-invariant and $f$ is bounded. And this proves that $S^{*} \mu$ is absolutely continuous with respect to $\mu$. So, as Fubuni's theorem proves that it is $\sigma$-additive, $S^{*} \mu$ is a finite measure on $\mathbf{X}$.

Moreover, we saw in Lemma 2.4 that for all non negative borelian function $f$ on $\mathbf{X}, \int S R f \mathrm{~d} \mu=\int f \mathrm{~d} \mu$ and this proves that $R^{*} S^{*} \mu=\mu$.

Then, we need to prove that $S^{*} \mu(\mathbf{X})>0$. But, for all $x \in \mathbf{X}$,

$$
P^{k} S(1)(x)=\mathbb{E}_{x} S 1\left(X_{k}\right) \geqslant \mathbb{P}_{x}(\{\tau=k+1\})
$$


So, $\sum_{k=0}^{n-1} P^{k} S(1) \geqslant \mathbb{P}_{x}(\{\tau \leqslant n+1\})$. And, as $\mu$ is $P$-invariant, taking the integral on both sides, we get that,

$$
n S^{*} \mu(X) \geqslant \int_{x \in \mathbf{X}} \mathbb{P}_{x}(\{\tau \leqslant n\}) \mathrm{d} \mu(x) .
$$

Finally, we use that for $\mu$-a.e. $x \in \mathbf{X}, \lim _{n} \mathbb{P}_{x}(\tau \leqslant n)=1$ and the dominated convergence theorem, tells us that $0<\mu(\mathbf{X}) \leqslant \lim n S^{*} \mu(\mathbf{X})$, so $S^{*} \mu(\mathbf{X})>0$.

Lemma 2.7. Let $\nu$ be a non-zero $Q$-invariant borelian measure on $\mathbf{X}$. Then, $R^{*} \nu$ is a non zero $P$-invariant measure on $\mathbf{X}$.

Moreover, $S^{*} R^{*} \nu=\nu$ and $\nu$ is absolutely continuous with respect to $R^{*} \nu$.

Finally, if $Q R(1)$ is bounded on $\mathbf{X}$, then $R^{*} \nu$ is a finite measure if and only if $\nu$ is.

Remark 2.8. The technical assumption $Q R 1$ bounded on $\mathbf{X}$ is reasonable.

More specifically, using the same notations as in Remark 2.2, we call $\mathbf{Y}$ linearily recurrent if $\sup _{y \in \mathbf{Y}} \mathbb{E}_{y} \tau_{\mathbf{Y}}$ is finite.

In this case, $R 1(x)=\mathbb{E}_{x} \tau_{\mathbf{Y}}$ and $Q R 1(x)=\mathbb{E}_{x} R 1\left(X_{\tau_{\mathbf{Y}}}\right) \leqslant \sup _{y \in \mathbf{Y}} \mathbb{E}_{y} \tau_{\mathbf{Y}}$ since for any $x \in \mathbf{X}, \mathbb{P}_{x}\left(X_{\tau_{\mathbf{Y}}} \in \mathbf{Y}\right)=1$ be definition of $\tau_{\mathbf{Y}}$.

Proof. To prove that $R^{*} \mu$ is a measure, one just have to prove that it is $\sigma$-additive.

Let $\left(A_{n}\right)$ be a sequence of pairwise disjoint borelian subsets of $\mathbf{X}$ and $n \in \mathbb{N}$. As $R$ is a linear operator, we have that $\int R\left(\mathbf{1}_{\cup_{k=0}^{n} A_{k}}\right) \mathrm{d} \nu=\sum_{k=0}^{n} \int R \mathbf{1}_{A_{k}} \mathrm{~d} \nu$, thus, $R^{*} \nu$ is finitely additive. But, according to the monotone convergence theorem, the left side of this equation converges to $\int R\left(\mathbf{1}_{\cup A_{n}}\right) \mathrm{d} \nu$ and this finishes the proof that $R^{*} \nu$ is $\sigma$-additive.

Moreover, for all non negative $f \in \mathcal{B}(\mathbf{X}), f \leqslant R f$, so $\nu(f) \leqslant \nu(R f)$ and $\nu$ is absolutely continuous with respect to $R^{*} \nu$ and $R^{*} \nu(X)>0$.

Then, Proposition 2.3 shows that for any positive borelian function $f, R f+Q f=f+R P f$. Applying this to $f=\mathbf{1}_{A}$ for some borelian set $A$, and taking the integral over $\nu$, we get that $\int R \mathbf{1}_{A}+Q \mathbf{1}_{A} \mathrm{~d} \nu=\int \mathbf{1}_{A}+R P \mathbf{1}_{A} \mathrm{~d} \nu$. But, $\nu$ is $Q$-invariant so if $\nu(A)$ is finite, we get that $\int R \mathbf{1}_{A} \mathrm{~d} \nu=\int R P \mathbf{1}_{A} \mathrm{~d} \nu$. If $\nu(A)$ is infinite, the result still holds since in this case, $\int R \mathbf{1}_{A} \mathrm{~d} \nu=\nu(A)=Q^{*} \nu(A)=\int R P \mathbf{1}_{A} \mathrm{~d} \nu=+\infty$. Thus, for any borelian set $A$, $R^{*} \nu(A)=P^{*} R^{*} \nu(A)$ that is to say, $R^{*} \nu$ is $P$-invariant.

As $R S=Q$ and $\nu$ is $Q$-invariant, we directly have that $S^{*} R^{*} \nu=\nu$.

For the last point, assume that $Q R(1)$ is a bounded function on $\mathbf{X}$.

If $R^{*} \nu$ is finite, then so is $\nu$ since $\nu(\mathbf{X}) \leqslant R^{*} \nu(\mathbf{X})$.

Assume that $\nu$ is finite. Then according to Chacon-Ornstein's ergodic theorem (see [5], Chap. 3 Thm. 3.4), there exist a $Q$-invariant non negative borelian function $g^{*}$ such that $\int g^{*} \mathrm{~d} \nu=\int R 1 \mathrm{~d} \nu$ and for $\nu$-almost every $x \in \mathbf{X}$,

$$
\frac{1}{n} \sum_{k=0}^{n-1} Q^{k} R 1(x) \rightarrow g^{*}(x)
$$

And, since $Q R$ is bounded on $\mathbf{X}$ and $R 1(x)=\mathbb{E}_{x} \tau$ is finite, we get that $g^{*}(x) \leqslant\|Q R\|_{\infty}$ for $\nu-$ a.e $x \in \mathbf{X}$. So, $g \in \mathrm{L}^{\infty}(\mathbf{X}, \nu) \subset \mathrm{L}^{1}(\mathbf{X}, \nu)$ since $\nu(\mathbf{X})<+\infty$ and $\int R 1 \mathrm{~d} \nu \leqslant\|Q R\|_{\infty} \nu(X)<+\infty$.

We saw in the previous lemmas that $R$ and $S$ act on invariant measure. As they are linear operators and the set of invariant measures is convex, next proposition shows that they also preserve the ergodic measures (in some sense since they do not preserve probability measures).

Corollary 2.9. Let $\left(X_{n}\right)$ be a Markov chain on a complete separable metric space $\mathbf{X}$ and $\tau$ a $\theta$-compatible stopping time such that for any $x \in \mathbf{X}, \mathbb{E}_{x} \tau$ is finite. Define $P, Q, R$ and $S$ as previously and assume that $Q R 1$ is bounded on $\mathbf{X}$.

Then, $S^{*}$ and $R^{*}$ are linear bijections between the $P$-invariant finite measures and the $Q$-invariant ones which preserve ergodicity and are inverse of each other. 
Proof. We already saw in Lemmas 2.6 and 2.7 that $S^{*}$ (resp. $R^{*}$ ) maps the $P$-invariant (resp. $Q$-invariant) finite non zero measures onto the $Q$-invariant (resp. $P$-invariant) ones and that they are inverse of each-other.

Thus, it remains to prove that the image by $S^{*}$ or $R^{*}$ of an ergodic measure still is ergodic. To do so, we use the linearity of $S^{*}$ and $R^{*}$ and that ergodic probability measures are extreme points of the set of invariant probability measures for a Markov chain in a complete separable metric space.

Let $\mu$ be a $P$-ergodic finite non zero measure. We assume without any loss of generality that $\mu$ is a probability measure. We saw in Lemma 2.6 that $S^{*} \mu$ is a $Q$-invariant non zero finite measure.

Assume that $S^{*} \mu=S^{*} \mu(\mathbf{X})\left(t \nu_{1}+(1-t) \nu_{2}\right)$ where $\nu_{1}$ and $\nu_{2}$ are two $Q$-invariant probability measures and $t \in[0,1]$.

Then, we get that $\mu=R^{*} S^{*} \mu=S^{*} \mu(X)\left(t R^{*} \nu_{1}+(1-t) R^{*} \nu_{2}\right)$. But $\mu$ is ergodic, so $\frac{1}{R^{*} \nu_{1}(\mathbf{X})} R^{*} \nu_{1}=$ $\frac{1}{R^{*} \nu_{2}(\mathbf{X})} R^{*} \nu_{2}$. And applyting $S^{*}$ again, we obtain that $\nu_{1}=\nu_{2}$, hence, $S^{*} \mu$ is $Q$-ergodic.

The same proof holds to show that if $\nu$ is $Q$-ergodic, then $R^{*} \nu$ is $P$-ergodic.

\section{INDUCTION AND THE RENEWAL THEOREM}

In this section, we use the renewal theorem on $\mathbb{R}$ to prove a "stopped renewal theorem" in Corollary 3.6.

Let $\rho$ be a borelian probability measure on $\mathbb{R}$ and define a random walk on $\mathbb{R}$ starting at $x \in \mathbb{R}$ by

$$
\left\{\begin{array}{ccc}
X_{0} & = & x \\
X_{n+1} & =X_{n}+Y_{n+1}
\end{array}\right.
$$

where $\left(Y_{n}\right)$ has law $\rho^{\otimes \mathbb{N}}$.

We assume that $\rho$ has a moment of order 1 and a negative drift $\lambda=\int_{\mathbb{R}} y \mathrm{~d} \rho(y)<0$.

In particular, for $\rho^{\otimes \mathbb{N}}$-a.e $\left(Y_{n}\right) \in \mathbb{R}^{\mathbb{N}}, \sum_{k=0}^{n} Y_{k}$ converges to $-\infty$.

We denote by $P$ the Markov operator associated to $\rho$. This is the operator defined for any bounded borelian function $f$ on $\mathbb{R}$ and any $x \in \mathbb{R}$ by

$$
P f(x)=\int_{\mathbb{R}} f(x+y) \mathrm{d} \rho(y)
$$

Let $\tau$ be the time of first return to $]-\infty, 0]$ :

$$
\left.\left.\tau\left(\left(X_{n}\right)\right)=\inf \left\{n \in \mathbb{N}^{*}, X_{n} \in\right]-\infty, 0\right]\right\}
$$

This is a $\vartheta$-compatible stopping time and our assumption on $\rho$ implies (see P1 in Sect. 18 of [9]) that for any $x \in \mathbb{R}$,

$$
\mathbb{E}_{x} \tau<+\infty
$$

In this section, we are interested in the operator $R$ defined as in Section 2 for any non negative borelian function $f$ on $\mathbb{R}$ and any $x \in \mathbb{R}$ by

$$
R f(x):=\mathbb{E}_{x} \sum_{k=0}^{\tau-1} f\left(X_{k}\right)
$$

The study of this operator is very close from renewal theory: indeed, if $\rho\left(\mathbb{R}_{+}\right)=0$ and $f$ is null on $\mathbb{R}_{-}^{*}$, then for any $x \in \mathbb{R}$,

$$
R f(x)=\sum_{n=0}^{+\infty} P^{n} f(x)
$$

Therefore, we make the following definition that is usual in renewal theory:

Definition 3.1. Let $f$ be a borelian function on $\mathbb{R}$. We say that $f$ is directly Riemann-integrable if

$$
\lim _{h \rightarrow 0^{+}} h \sum_{n \in \mathbb{Z}} \inf _{x \in[n h,(n+1) h]}|f(x)|=\lim _{h \rightarrow 0^{+}} h \sum_{n \in \mathbb{Z}} \sup _{x \in[n h,(n+1) h]}|f(x)|<+\infty
$$

In the sequel, we will use the following characterisation. 
Lemma 3.2 (Lebesgue's criterion for Riemann-integrability). Let $f$ be a bounded function on $\mathbb{R}$.

Then, $f$ is directly Riemann integrable if and only if it is a.e. continuous and for some $h \in \mathbb{R}_{+}^{*}$,

$$
\sum_{n \in \mathbb{Z}} \sup _{x \in[n h,(n+1) h]}|f(x)|<+\infty .
$$

In the next three lemmas, we are going to prove that, denoting by $R, S$ the operators defined as in Section 2 and associated to $\tau$, for any directly Riemann-integrable function $f, S R f$ is also directly Riemann-integrable.

Lemma 3.3. Let $\rho$ be a borelian probability measure on $\mathbb{R}$ having a moment of order 1 and a negative drift $\lambda=\int_{\mathbb{R}} y \mathrm{~d} \rho(y)<0$.

Note $\tau$ the time of first return to $]-\infty, 0]$ and $R$ the associated operator defined as in Section 2.

Then, for any directly Riemann-integrable function $f$ on $\mathbb{R}$, the function $R f$ is bounded on $\mathbb{R}$.

Proof. To prove this proposition, we are going to use the classical renewal theorem. Indeed, for any $x \in \mathbb{R}$ we have that

$$
|R f(x)| \leqslant R|f|(x)=\mathbb{E}_{x} \sum_{k=0}^{\tau-1}\left|f\left(X_{k}\right)\right| \leqslant \mathbb{E}_{x} \sum_{n=0}^{+\infty}\left|f\left(X_{n}\right)\right|=\sum_{n=0}^{+\infty} P^{n}|f|(x) .
$$

But, if the measure is non-lattice ${ }^{2}$, according to the renewal theorem (see [2]), we have that

$$
\lim _{x \rightarrow-\infty} \sum_{n=0}^{+\infty} P^{n}|f|(x)=0 \text { and } \lim _{x \rightarrow+\infty} \sum_{n=0}^{+\infty} P^{n}|f|(x)=\frac{-1}{\lambda} \int_{\mathbb{R}}|f| \mathrm{d} \nu
$$

and this proves our lemma in the non-lattice case. The same kind of arguments holds in the lattice case and allows us to conclude.

Lemma 3.4. Let $\rho$ be a borelian probability measure on $\mathbb{R}$ having a moment of order 1 and a negative drift $\lambda=\int_{\mathbb{R}} y \mathrm{~d} \rho(y)<0$.

Then, for any directly-Riemann integrable function $f$ on $\mathbb{R}$ that is null on $\mathbb{R}_{-}$, we have that

$$
\sum_{n \in \mathbb{Z}} \sup _{x \in[n,(n+1)]}|S R f(x)|<+\infty .
$$

Proof. First, according to Lemma 3.3, $R f$ is bounded and for any $x \in \mathbb{R}$,

$$
\left.\left.|S R f(x)| \leqslant\|R f\|_{\infty} S 1(x)=\|R f\|_{\infty} \rho(]-\infty,-x\right]\right) .
$$

So, as the function $(x \mapsto \rho(]-\infty,-x]))$ is decreasing, we have that

$$
\left.\left.\sum_{n \in \mathbb{N}} \sup _{x \in[n,(n+1)]}|S R f(x)| \leqslant\|R f\|_{\infty} \sum_{n \in \mathbb{N}} \rho(]-\infty,-n\right]\right) .
$$

Moreover, using that

$$
\left.\left.\rho(]-\infty,-n])=\sum_{m=n}^{+\infty} \rho(]-m-1,-m\right]\right) .
$$

We have that

$$
\begin{aligned}
\left.\left.\sum_{n \in \mathbb{N}} \sum_{m=n}^{+\infty} \rho(]-(m+1),-m\right]\right) & \left.\left.\left.\left.=\sum_{m \in \mathbb{N}}(m+1) \rho(]-(m+1),-m\right]\right)=\rho\left(\mathbb{R}_{-}\right)+\sum_{m \in \mathbb{N}} m \rho(]-(m+1),-m\right]\right) \\
& \leqslant 1+\sum_{m \in \mathbb{N}} \int_{-(m+1)}^{-m}|y| \mathrm{d} \rho(y) \leqslant 1+\int_{\mathbb{R}}|y| \mathrm{d} \rho(y) .
\end{aligned}
$$

\footnotetext{
${ }^{2}$ For any $\alpha \in \mathbb{R}, \rho(\alpha \mathbb{Z})<1$.
} 
And this proves that

$$
\sum_{n \in \mathbb{N}} \sup _{x \in[n, n+1]}|S R f(x)|<+\infty
$$

We now have to control the sum over $\mathbb{Z}_{-}$.

Since for any $x \in \mathbb{R}_{-}, f(x)=0$, we have, using Markov's property, that for any $x \in \mathbb{R}$,

$$
S R f(x)=\mathbb{E}_{x} \mathbf{1}_{\{\tau=1\}} \sum_{k=1}^{\tau^{2}-1} f\left(X_{k}\right)=\mathbb{E}_{x} \mathbf{1}_{\left\{\tau=1, \tau^{2}>2\right\}} \sum_{k=2}^{\tau^{2}-1} f\left(X_{k}\right)=\mathbb{E}_{x} \mathbf{1}_{\left\{\tau=1, \tau^{2}>2\right\}} R f\left(X_{2}\right)
$$

So,

$$
|S R f(x)| \leqslant \mathbb{E}_{x} \mathbf{1}_{\{\tau=1\} \cap\left\{\tau^{2}>2\right\}}\left|R f\left(X_{2}\right)\right| \leqslant\|R f\|_{\infty} \mathbb{P}_{x}\left(\tau=1, \tau^{2}>2\right)
$$

But,

$$
\mathbb{P}_{x}\left(\tau=1, \tau^{2}>2\right)=\int_{\mathbb{R}^{2}} \mathbf{1}_{\left\{x+y_{1} \leqslant 0, x+y_{1}+y_{2}>0\right\}} \mathrm{d} \rho\left(y_{1}\right) \mathrm{d} \rho\left(y_{2}\right) \leqslant \rho^{* 2}(]-x,+\infty[)
$$

So, using the fact that the function $\left(x \mapsto \rho^{* 2}(]-x,+\infty[)\right)$ is non-decreasing, we have that

$$
\begin{array}{r}
\left.\left.\sum_{n=-\infty}^{0} \sup _{x \in[n-1, n]}|S R f(x)| \leqslant\|R f\|_{\infty} \sum_{n=0}^{+\infty} \rho^{* 2}(] n,+\infty[)=\|R f\|_{\infty} \sum_{n=0}^{+\infty} \sum_{m=n}^{+\infty} \rho^{* 2}(] m, m+1\right]\right) \\
\left.\left.\leqslant\|R f\|_{\infty} \sum_{m=0}^{+\infty}(m+1) \rho^{* 2}(] m, m+1\right]\right) \leqslant\|R f\|_{\infty}\left(1+\int_{0}^{+\infty} y \mathrm{~d} \rho(y)\right)
\end{array}
$$

And this finishes the proof of the lemma.

Lemma 3.5. Let $\rho$ be a borelian probability measure that is the sum of an absolutely continuous measure $\rho_{1}$ and a discrete measure $\rho_{2}$.

Then, for any bounded and a.e. continuous function $f$ on $\mathbb{R}, R f$ and $S f$ are also a.e. continuous.

Proof. For any $x \in \mathbb{R}$, we have that

$$
\begin{aligned}
S f(x) & =\int_{\mathbb{R}} f(x+y) \mathbf{1}_{\{x+y \leqslant 0\}} \mathrm{d} \rho(y) \\
& =\int_{\mathbb{R}} f(x+y) \mathbf{1}_{\{x+y \leqslant 0\}} \varphi(y) \mathrm{d} y+\sum_{y \in \operatorname{supp} \rho_{2}} f(x+y) \mathbf{1}_{\{x+y \leqslant 0\}} \rho_{2}(y)
\end{aligned}
$$

Where we denoted $\varphi$ the density of $\rho_{1}$ and we used the fact that $\rho_{2}$ is atomic.

But, $x \mapsto f(x) \mathbf{1}_{\{x \leqslant 0\}}$ is bounded and $\varphi$ is integrable so the function

$$
x \mapsto \int_{\mathbb{R}} f(x+y) \mathbf{1}_{\{x+y \leqslant 0\}} \varphi(y) \mathrm{d} y
$$

is continuous on $\mathbb{R}$ (as a convolution product of an integrable and a bounded function).

And, as supp $\rho_{2}$ is denumerable and $f$ is a.e. continuous, the function

$$
x \mapsto \sum_{y \in \operatorname{supp} \rho_{2}} f(x+y) \mathbf{1}_{\{x+y \leqslant 0\}} \rho_{2}(y)
$$

still is a.e. continuous.

This proves that $S f$ is a.e. continuous. 
To prove that $R f$ is a.e. continuous, note that for any $x \in \mathbb{R}$, we can write

$$
R f(x)=\sum_{n=0}^{+\infty} \mathbb{E}_{x} f\left(X_{n}\right) \mathbf{1}_{\{\tau>n\}}
$$

Moreover, we have that

$$
\left|\mathbb{E}_{x} f\left(X_{n}\right) \mathbf{1}_{\{\tau>n\}}\right| \leqslant\|f\|_{\infty} \mathbb{P}_{x}(\tau>n)
$$

so, using the fact that the function $\left(x \mapsto \mathbb{P}_{x}(\tau>n)\right)$ is non-decreasing, we have that for any $x_{0} \in \mathbb{R}$ and any $\left.x \in]-\infty, x_{0}\right]$,

$$
\left|\mathbb{E}_{x} f\left(X_{n}\right) \mathbf{1}_{\{\tau>n\}}\right| \leqslant\|f\|_{\infty} \mathbb{P}_{x_{0}}(\tau>n) .
$$

So the convergence in the series is uniform on every compact subset of $\mathbb{R}$ since we already saw that

$$
\sum_{n} \mathbb{P}_{x_{0}}(\tau>n)=\mathbb{E}_{x_{0}} \tau<+\infty .
$$

Therefore, the set of continuity points of $R f$ contains the intersection of the sets of continuity points of the functions $\left(x \mapsto \mathbb{E}_{x} f\left(X_{n}\right) \mathbf{1}_{\{\tau>n\}}\right)$ for $n \in \mathbb{N}$. Moreover, for any $x \in \mathbb{R}$ and any $n \in \mathbb{N}$,

$$
\mathbb{E}_{x} f\left(X_{n}\right) \mathbf{1}_{\{\tau>n\}}=\int_{\mathbb{R}^{n}} f\left(x+y_{1}+\ldots+y_{n}\right) \mathbf{1}_{\forall k \in[1, n], x+y_{1}+\ldots+y_{k}>0} \mathrm{~d} \rho^{\otimes n}\left(\left(y_{i}\right)\right) .
$$

And we can see that this function is a.e. continuous by using the same kind of arguments than in the proof of the a.e. continuity of $S f$.

Corollary 3.6. Let $\rho$ be a borelian probability measure on $\mathbb{R}$ having a moment of order 1 and a negative drift $\lambda=\int_{\mathbb{R}} y \mathrm{~d} \rho(y)<0$. Assume that $\rho$ is non lattice ${ }^{3}$ and the sum of an absolutely continuous measure and of a discrete one.

Note $\tau$ the time of first return to $]-\infty, 0]$ and denote $R$ and $S$ the Markov operators defined for any borelian bounded function $f$ on $\mathbb{R}$ and any $x \in \mathbb{R}$ by

$$
R f(x)=\mathbb{E}_{x} \sum_{k=0}^{\tau-1} f\left(X_{k}\right) \text { and } S f(x)=\int_{\{\tau=1\}} f\left(X_{1}\right) \mathrm{d} \mathbb{P}_{x}\left(\left(X_{n}\right)\right) .
$$

Then, for any directly Riemann-integrable function $f$ on $\mathbb{R}$,

$$
\lim _{x \rightarrow+\infty} R f(x)=\frac{-1}{\lambda} \int_{\mathbb{R}}\left(I_{d}-S R\right) f(u) \mathrm{d} u .
$$

Proof. By definition of the operator $R$, we shall assume without any loss of generality that $f=0$ on $\mathbb{R}_{-}$.

According to the previous lemmas $(3.2,3.3,3.4$ and 3.5$)$ we have that $f-S R f$ is directly Riemann-integrable. Thus, we can apply the renewal theorem to get that

$$
\lim _{x \rightarrow+\infty} \sum_{n=0}^{+\infty} P^{n}\left(I_{d}-S R\right) f(x)=\frac{-1}{\lambda} \int_{\mathbb{R}}\left(I_{d}-S R\right) f(u) \mathrm{d} u .
$$

But, for any $n \in \mathbb{N}$,

$$
\sum_{k=0}^{n-1} P^{k}\left(I_{d}-S R\right) f(x)=\sum_{k=0}^{n-1} P^{k}\left(I_{d}-P\right) R f(x)=R f(x)-P^{n} R f(x)
$$

\footnotetext{
${ }^{3}$ For any $\alpha \in \mathbb{R}, \rho(\alpha \mathbb{Z})<1$.
} 
and, as $f$ is null on $\mathbb{R}_{-}$and $R f$ is bounded according to Lemma 3.3, we have that

$$
\lim _{n \rightarrow+\infty} P^{n} R f(x)=0
$$

Thus, for any $x \in \mathbb{R}$,

$$
R f(x)=\sum_{n=0}^{+\infty} P^{n}\left(I_{d}-S R\right) f(x)
$$

Which is what we intended to prove.

\section{Application to the Relfected RAndom Walk on $\mathbb{R}_{+}$}

In this section, we use the previous results for the stopped renewal theorem to study the regularity at infinity of the solution of Poisson's equation for the reflected random walk on $\mathbb{R}_{+}$.

Let $\rho$ be a probability measure on $\mathbb{R}$ such that $\int_{\mathbb{R}}|y| \rho(\mathrm{d} y)$ is finite, $\int_{\mathbb{R}} y \rho(\mathrm{d} y)<0$ and $\rho\left(\mathbb{R}_{+}^{*}\right) \neq 0$.

These last two asumptions means that for $\rho^{\otimes \mathbb{N}}$-a.e $\left(Y_{n}\right) \in \mathbb{R}^{\mathbb{N}}, \sum_{k=0}^{n} Y_{k}$ converges to $-\infty$ but for any fixed $M \in \mathbb{R}, \rho^{\otimes \mathbb{N}}\left(\left\{\left(Y_{n}\right) \in \mathbb{R}^{\mathbb{N}} ; \exists n \in \mathbb{N} \sum_{k=0}^{n-1} Y_{k} \geqslant M\right\}\right)>0$.

Let $\left(Y_{n}\right)_{n \in \mathbb{N}}$ be an iid seqence of random variables of law $\rho$. We define the reflected random walk starting at $x$ on $\mathbb{R}_{+}$by

$$
\left\{\begin{aligned}
X_{0} & =x \\
\text { for all } n \in \mathbb{N}, X_{n+1} & =\left|X_{n}+Y_{n+1}\right|
\end{aligned}\right.
$$

Defined like this, $\left(X_{n}, Y_{n}\right)_{n \in \mathbb{N}}$ is a Markov chain on $\mathbb{R}_{+} \times \mathbb{R}$. As Peigné and Woess in [8], we define a stopping time which we call the time of first reflection by

$$
\tau\left(\left(X_{n}, Y_{n}\right)\right)=\inf \left\{n \in \mathbb{N}^{*} ; X_{n-1}+Y_{n}<0\right\}
$$

We see that $\tau$ is $\theta$-compatible since it is the time of first return in $\mathbb{R}_{-}^{*}$ for the unreflected random walk on $\mathbb{R}$ driven by $\rho$.

Since $\int y \mathrm{~d} \rho(y)<0, \sum_{k=0}^{n-1} Y_{k} \underset{n \rightarrow \infty}{\longrightarrow}-\infty$ a.e., and $\tau_{x}^{n}$ is well defined (finite almost everywhere for all positive real number $x$ ).

We define the operator $P, Q, R$ and $S$ as in Section 2 .

Remark 4.1. For a borelian non negative function $f$ on $\mathbb{R}_{+} \times \mathbb{R}$ we defined $P(f)(x, y)=\mathbb{E}_{(x, y)} f\left(X_{1}, Y_{1}\right)$. But, since $X_{1}$ and $Y_{1}$ are independant of $Y_{0}$, we have that $P(f)(x, y)=\mathbb{E}_{x} f\left(X_{1}, Y_{1}\right)=\int_{\mathbb{R}} f(|x+y|, y) \rho(\mathrm{d} y)$. In particular, if $f$ itself does not depend of it's second argument, we have $P(f)(x, y)=\mathbb{E}_{(x, y)} f\left(X_{1}\right)=\mathbb{E}_{x} f\left(X_{1}\right)$ and we find the usual Markov operator associated to $\left(X_{n}\right)$.

The same argument applies to $Q, R$ and $S$ (defined as in Sect. 2)

Those considerations are just made to prove that $\tau$ is $\theta$-compatible so we can apply the results of the previous sections, but we can anyway "forget" about the second variable.

From now on, we identify functions on $\mathbb{R}_{+}$and functions on $\mathbb{R}_{+} \times \mathbb{R}$ which don't depend on their second variable and we make the abuse of notations that come with this identification.

As we will need some regularity assumption on $\rho$, we make the following

Definition 4.2 (Spread-out probability measure on $\mathbb{R}$ ). We say that a probability measure $\rho$ on $\mathbb{R}$ is spread-out if there exist $m \in \mathbb{N}^{*}$ such that $\rho^{* m}$ is not singular with respect to Lebesgue's measure on $\mathbb{R}$.

First, we have the following 
Theorem 4.3 (Leguesdron [6], Peigné-Woess [8]). Let $\rho$ be a spread-out probability measure on $\mathbb{R}$ having an moment of order 1 and a negative drift $\lambda=\int_{\mathbb{R}} y \mathrm{~d} \rho(y)$. Consider $\left(X_{n}\right)$, the reflected random walk associated to $\rho$.

There exist a unique $P$-invariant probability measure $\nu$ on $\mathbb{R}_{+}$. Moreover, if $\rho\left(\mathbb{R}_{+}^{*}\right)=0$, then $\operatorname{supp} \nu=[0, M]$, else $\operatorname{supp} \nu=\mathbb{R}_{+}$where $M=-\inf \operatorname{supp}(\rho)$ (which may be infinite).

Finally, the reflected random walk is topologically irreducible on $\operatorname{supp} \nu$.

To solve Poisson's equation, we are going to use the theory of petite sets developed by Glynn, Meyn and Tweedie (see $[3,7]$ ).

Definition 4.4. Let $\left(X_{n}\right)$ be a Markov chain on a standard Borel space $\mathbf{X}$ and $P$ the associated Markov operator. Let $a$ be a probability measure on $\mathbb{N}$. A set $C \subset \mathbf{X}$ is called $\nu$-petite where $\nu$ is a non trivial measure on $\mathbf{X}$ if for any borelian subset $A$ of $\mathbf{X}$ and any $x \in C$,

$$
\sum_{n \in \mathbb{N}} a_{n} P^{n}\left(\mathbf{1}_{A}\right)(x) \geqslant \nu(A)
$$

Proposition 4.5. Let $\rho$ be a spread out probability measure on $\mathbb{R}$ having a moment of order 1 and such that $\rho\left(\mathbb{R}_{+}^{*}\right)>0$.

Then the reflected random walk on $\mathbb{R}_{+}$defined by $\rho$ is irreducible and every compact set is petite.

Proof. As we already saw in Theorem 4.3, if $\int|y| \rho(\mathrm{d} y)$ is finite, reflected random walk is open-set irreducible on $\mathbb{R}_{+}$.

Therefore, if we prove that $\left(X_{n}\right)$ is a T-chain (see [7]), using the first point of Theorem 6.0.1 in [7] we will get that the chain is $m$-irreducible and then, using the second point of this theorem, we will get that every compact set is petite.

We need to find $\left(a_{n}\right) \in[0,1]^{\mathbb{N}}$ such that $\sum_{n} a_{n}=1$ and a substochastic transition kernel $T$ such that $\forall x \in \mathbf{X} T(1)(x)>0$, for any borelian set $A, T\left(\mathbf{1}_{A}\right)$ is lower semicontinuous and

$$
\forall x \in \mathbf{X} \forall A \in \mathcal{B}(\mathbf{X}) \sum_{n=0}^{+\infty} a_{n} P^{n}\left(\mathbf{1}_{A}\right)(x) \geqslant T\left(\mathbf{1}_{A}\right)(x) .
$$

We assume without any loss of generality that $\rho$ is compactly supported and we denote by $M \in \mathbb{R}_{+}^{*}$ some real number such that $\operatorname{supp} \rho \subset[-M,+\infty[)$.

Let $m$ be such that $\rho^{* m}$ is not singular with respect to Lebesgue's measure. We denote by $\psi$ it's RadonNikodym's derivative.

Let $\varepsilon \in \mathbb{R}_{+}^{*}$ such that $\rho\left(\left[\varepsilon,+\infty[)>0\right.\right.$ (such $\varepsilon$ exists since $\rho\left(\mathbb{R}_{+}^{*}\right)>0$ ). We denote by $\rho_{\varepsilon}$ the measure defined by $\rho_{\varepsilon}(A)=\rho\left(A \cap\left[\varepsilon,+\infty[), X_{n}^{\varepsilon}\right.\right.$ the random walk assoicated to $\rho_{\varepsilon}$ and $P_{\varepsilon}$ the submarkov operator associated to $\rho_{\varepsilon}$ that is to say: $P_{\varepsilon}(f)(x)=\int_{\mathbb{R}} f(|x+y|) \mathrm{d} \rho_{\varepsilon}(y)=\int_{\varepsilon}^{+\infty} f(x+y) \mathrm{d} \rho(y)$.

The main idea of this proof is that, using $\rho_{\varepsilon}$, we can "escape" any compact set, and in particular, if we walk for a long enough time $N$ with $\rho_{\varepsilon}(N$ such that $N \varepsilon \geqslant m M)$, we can be sure that the time of first reflection for the walk starting at $X_{N}^{\varepsilon}$ is greater than $m$. And thus, we can use the hypothesis that $\rho$ is spread out.

More precisely, if $f$ is a non negative borelian function on $\mathbb{R}_{+}$and $N \in \mathbb{N}$ is such that $N \varepsilon \geqslant m M$, then,

$$
\begin{aligned}
P^{m+N} f(x) & \geqslant \int_{\mathbb{R}^{m+N}} f\left(|| x+y_{1}\left|+\ldots+y_{m+N}\right|\right) \mathrm{d} \rho_{\varepsilon}^{\otimes N} \otimes \mathrm{d} \rho^{\otimes m}\left(\left(y_{i}\right)\right) \\
& \geqslant \int_{\mathbb{R}^{m+N}} f\left(x+y_{1}+\ldots+y_{m+N}\right) \mathrm{d} \rho_{\varepsilon}^{\otimes N} \otimes \mathrm{d} \rho^{\otimes m}\left(\left(y_{i}\right)\right) \\
& \geqslant \int_{\mathbb{R}^{2}} f(x+v+u) \mathrm{d} \rho_{\varepsilon}^{* N}(v) \mathrm{d} \rho^{* m}(u) \geqslant \int_{\mathbb{R}^{2}} f(x+v+u) \mathrm{d} \rho_{\varepsilon}^{* N}(v) \psi(u) \mathrm{d} u \\
& \geqslant \int_{\mathbb{R}} f(x+u) \int_{\mathbb{R}} \psi(u-v) \mathrm{d} \rho_{\varepsilon}^{* N}(v) \mathrm{d} u=\int_{\mathbb{R}} f(x+u) \psi_{1}(u) \mathrm{d} u .
\end{aligned}
$$


Where, for $u \in \mathbb{R}, \psi_{1}(u)=\int_{\mathbb{R}} \psi(u-v) \mathrm{d} \rho_{\varepsilon}^{* N}(v)$.

For $n \in \mathbb{N}$, let $\psi_{2}^{n}(u)=\min \left(n, \psi_{1}(u)\right) \in \mathrm{L}^{1} \cap \mathrm{L}^{\infty}$. By definition, $\psi_{2}^{n}$ is a non decreasing sequence of positive functions and using the monotone convergence theorem, we have that $\int \psi_{2}^{n}(u) \mathrm{d} u \underset{n \rightarrow+\infty}{\longrightarrow} \int \psi_{1} \mathrm{~d} u=\rho_{\varepsilon}(\mathbb{R})^{N}=$ $\rho\left(\left[\varepsilon,+\infty[)^{N} \in\right] 0,1\right]$. So there exist $n_{0} \in \mathbb{N}$ such that $\int \psi_{2}^{n_{0}}(u) \mathrm{d} u \neq 0$.

We denote $\psi_{2}^{n_{0}}=\psi_{2}$ and we resume our computations.

By construction,

$$
\begin{aligned}
P^{2 m+2 N} f(x) & \geqslant \int_{\mathbb{R}} P^{m+N} f(x+u) \psi_{2}(u) \mathrm{d} u \geqslant \int_{\mathbb{R}^{2}} f(x+u+v) \psi_{2}(v) \psi_{2}(u) \mathrm{d} u \mathrm{~d} v \\
& \geqslant \int_{\mathbb{R}} f(x+u)\left(\int_{\mathbb{R}} \psi_{2}(u-v) \psi_{2}(v) \mathrm{d} v\right) \mathrm{d} u=\int_{\mathbb{R}} f(x+u) \psi_{3}(u) \mathrm{d} u
\end{aligned}
$$

Where $\psi_{3}(u)=\int_{\mathbb{R}} \psi_{2}(u-v) \psi_{2}(v) \mathrm{d} v$.

But, $\psi_{3}$ is the convolution product of an integrable function and of a bounded one so it is continuous and $\int_{\mathbb{R}} \psi_{3}(u) \mathrm{d} u=\left(\int_{\mathbb{R}} \psi_{2}(u) \mathrm{d} u\right)^{2}>0$.

Let $\psi_{4}$ be a non zero non negative continuous function on $\mathbb{R}$ such that $\psi_{4} \leqslant \psi_{3}$ and $\operatorname{supp} \psi_{4}$ is compact.

We denote $T$ the operator defined for any borelian bounded function $f$ on $\mathbb{R}$ and any $x \in \mathbb{R}$ by

$$
T f(x)=\int_{\mathbb{R}} f(x+u) \psi_{4}(u) \mathrm{d} u
$$

Using the dominated convergence theorem we have that for any borelian and bounded function $f, T f$ is continuous. Moreover, for all $x \in \mathbb{R}_{+}, 1 \geqslant T 1(x)=\int_{\mathbb{R}} \psi_{4} \mathrm{~d} \lambda>0$.

And, we get that for every borelian non negative function $f$ and every $x$ in $\mathbb{R}_{+}$,

$$
P^{2 N+2 m} f(x) \geqslant T f(x)
$$

and this finishes the proof of the proposition.

Proposition 4.6. Let $\rho$ be a spread out probability measure on $\mathbb{R}$ having a moment of order 1 and such that $0<\int_{\mathbb{R}_{+}} y \mathrm{~d} \rho(y)<\int_{\mathbb{R}_{-}}(-y) \mathrm{d} \rho(y)$.

Then, there is a constant $C$ such that for any borelian and bounded fonction $f$ on $\mathbb{R}_{+}$, there is a function $g$ on $\mathbb{R}_{+}$such that

$$
f=g-P g+\int f \mathrm{~d} \nu
$$

And,

$$
\sup _{x \in \mathbb{R}} \frac{|g(x)|}{1+|x|} \leqslant C\left\|f-\int f \mathrm{~d} \nu\right\|_{\infty}
$$

Moreover, if $\rho$ is the sum of an absolutely continuous measure and of a discrete one and if $f$ is a.e. continuous, then so is $g$.

Proof. For any $x \in \mathbb{R}_{+}$, let $u(x)=1+\frac{x}{-\lambda}$ where we denoted $\lambda=\int_{\mathbb{R}} y \mathrm{~d} \rho(y)<0$.

Then,

$$
\begin{aligned}
P u(x) & =1+\int_{-\infty}^{-x} \frac{x+y}{\lambda} \mathrm{d} \rho(y)+\int_{-x}^{+\infty} \frac{-x-y}{\lambda} \mathrm{d} \rho(y) \\
& \left.\left.\left.\left.=1+\frac{x}{\lambda}(\rho(]-\infty,-x]\right)-\rho(]-x,+\infty\right]\right)\right)-\frac{1}{\lambda}\left(\int_{-x}^{+\infty} y \mathrm{~d} \rho(y)-\int_{-\infty}^{-x} y \mathrm{~d} \rho(y)\right) \\
& \left.\left.=u(x)-1+2 \frac{x}{\lambda} \rho(]-\infty,-x\right]\right)+\frac{2}{\lambda} \int_{-\infty}^{-x} y \mathrm{~d} \rho(y)
\end{aligned}
$$


Moreover,

$$
x \rho(]-\infty,-x])=\int_{-\infty}^{-x} x \mathrm{~d} \rho(y) \leqslant \int_{-\infty}^{-x}|y| \mathrm{d} \rho(y) .
$$

So, as $\rho$ has a moment of order 1 ,

$$
\lim _{x \rightarrow+\infty} P u(x)-u(x)=-1 .
$$

Thus, there are $x_{0} \in \mathbb{R}_{+}$and $b \in \mathbb{R}$ such that

$$
P u \leqslant u-\frac{1}{2}+b \mathbf{1}_{\left[0, x_{0}\right]} .
$$

Therefore, using Proposition 4.5 we can apply Theorem 2.3 in [3] and get a constant $C$ such that for any borelian bounded function $f$ on $\mathbb{R}_{+}$there is a borelian function $g$ such that

$$
\sup _{x} \frac{|g(x)|}{u(x)} \leqslant C\left\|f-\int f \mathrm{~d} \nu\right\|_{\infty}
$$

and

$$
f=g-P g+\int f \mathrm{~d} \nu .
$$

The fact that $g$ is a.e. continuous when $f$ is a.e. continuous is also proved since we have an explicit formula for the function $g$ given in [3] and using the same ideas as in the proof of Lemma 3.5.

From now on, the assumption on $\rho$ being only spread-out is not enough (since the stopped renewal theorem we have in this case doesn't hold for these probability measures) so we are going to ask that it is absolutely continuous instead.

Corollary 4.7. Let $\rho$ be an absolutely continuous probability measure on $\mathbb{R}$ having a moment of order 1 , a negative drift $\lambda=\int_{\mathbb{R}} y \mathrm{~d} \rho(y)<0$ and such that $\rho\left(\mathbb{R}_{+}^{*}\right) \neq 0$.

Then, for any directly Riemman-integrable function $f$ on $\mathbb{R}_{+}$such that $\int f \mathrm{~d} \nu=0$, there is a bounded and a.e. continuous function $g$ on $\mathbb{R}_{+}$such that

$$
f=g-P g \text { and } \lim _{x \rightarrow+\infty} g(x)=0 .
$$

Proof. According to the previous lemma, there is an a.e. continuous function $g$ on $\mathbb{R}_{+}$such that

$$
f=g-P g \text { and } \sup _{x \in \mathbb{R}_{+}} \frac{|g(x)|}{1+|x|} \leqslant C\|f\|_{\infty} .
$$

We denote $\tau$ the time of first reflection. This is the stopping time defined by

$$
\tau\left(\left(X_{n}\right)\right)=\inf \left\{n \in \mathbb{N} \mid X_{n+1}=-X_{n}-Y_{n}\right\} .
$$

Moreover, we denote by $R$ and $S$ the Markov operators associated to $\tau$ and defined as in Section 2 .

Note that for $x \geqslant 0$ and before the reflection, the walk is the same as the unreflected random walk. Therefore, as in Section 3 we have that for any $x \in \mathbb{R}_{+}$,

$$
\mathbb{E}_{x} \tau<+\infty
$$

The stopping time $\tau$ is $\vartheta$-compatible so, we can use the relations of Proposition 2.3 to get that for any $x \in \mathbb{R}_{+}$,

$$
R f(x)=R(g-P g)(x)=g(x)-Q g(x) .
$$


Moreover, we also have that $Q g(x)=R S g(x)$ so, we get that $g(x)=R(f+S g)(x)$. Then,

$$
|Q g(x)|=\left|\mathbb{E} g\left(X_{\tau}\right)\right| \leqslant C \mathbb{E}_{x} 1+X_{\tau}
$$

But, using that $0 \leqslant X_{\tau} \leqslant-Y_{\tau}$, we have that

$$
\mathbb{E}_{x} X_{\tau} \leqslant-\mathbb{E}_{x} Y_{\tau} \leqslant \int_{\mathbb{R}}|y| \mathrm{d} \rho(y)
$$

So the function $Q g$ is bounded on $\mathbb{R}_{+}$and as $R f$ is bounded on $\mathbb{R}_{+}$(according to Lem. 3.3 that we can use for $x \geqslant 0$ since the operator $R$ for the reflected random walk and for the unreflected one are the same), this proves that $g$ is bounded on $\mathbb{R}_{+}$.

Thus, using Lemmas 3.4 and 3.5, we get that $S g$ is directly Riemann-integrable.

Therefore, we can apply Corollary 3.6 to the function $f+S g$ to get that $g=R(f+S g)$ has a limit $l$ at infinity. Noting $g_{1}=g-l$ we have that $g_{1}$ is a.e. continuous, bounded,

$$
g_{1}-P g_{1}=f \text { and } \lim _{x \rightarrow+\infty} g_{1}(x)=0
$$

And this is finally what we intended to prove.

Theorem 4.8 (Non-concentration inequality). Under the assumptions of Corollary 4.7.

For any directly Riemann-integrable function $f$ on $\mathbb{R}_{+}$such that $\int f \mathrm{~d} \nu=0$, any $\left.\left.\varepsilon \in\right] 0,1\right]$, any $x \in \mathbb{R}_{+}$and any $n \in \mathbb{N}^{*}$,

$$
\mathbb{P}_{x}\left(\left\{\left|\frac{1}{n} \sum_{k=0}^{n-1} f\left(X_{k}\right)\right| \geqslant \varepsilon\|g\|_{\infty}\right\}\right) \leqslant 3 \mathrm{e}^{-n \varepsilon^{2} / 8}
$$

where $g$ is the function given by Corollary 4.7 and such that $f=g-P g$.

In particular, for any $x \in \mathbb{R}_{+}$,

$$
\frac{1}{n} \sum_{k=0}^{n-1} f\left(X_{k}\right) \rightarrow 0 \mathbb{P}_{x} \text {-a.e. }
$$

Proof. To prove the result, we are going to use Azuma-Hoeffding's inequality.

First, we write $f=g-P g$ where $g$ is the function given by Corollary 4.7. Write, for any $n \in \mathbb{N}$,

$$
\frac{1}{n} \sum_{k=0}^{n-1} f\left(X_{k}\right)=\frac{1}{n} \sum_{k=0}^{n-1} g\left(X_{k+1}\right)-P g\left(X_{k}\right)+\frac{1}{n}\left(g\left(X_{0}\right)-g\left(X_{n}\right)\right)
$$

Thus,

$$
\frac{1}{n}\left|\sum_{k=0}^{n-1} g\left(X_{k+1}\right)-P g\left(X_{k}\right)\right| \geqslant \frac{1}{n}\left|\sum_{k=0}^{n-1} f\left(X_{k}\right)\right|-\frac{2}{n}\|g\|_{\infty}
$$

and so, using Azuma-Hoeffding's inequality, if $n \varepsilon>2$, we have that

$$
\begin{aligned}
I_{n}(x): & =\mathbb{P}_{x}\left(\frac{1}{n}\left|\sum_{k=0}^{n-1} f\left(X_{k}\right)\right|>\varepsilon\|g\|_{\infty}\right) \leqslant \mathbb{P}_{x}\left(\left|\sum_{k=0}^{n-1} g\left(X_{k+1}\right)-P g\left(X_{k}\right)\right| \geqslant(n \varepsilon-2)\|g\|_{\infty}\right) \\
& \leqslant 2 \exp \left(\frac{-(n \varepsilon-2)^{2}\|g\|_{\infty}^{2}}{2 n\left(2\|g\|_{\infty}\right)^{2}}\right)=2 \exp \left(-\frac{n \varepsilon^{2}}{8}+\frac{\varepsilon}{4}-\frac{1}{2 n}\right)
\end{aligned}
$$

and this last inequality is what we intended to prove since we took $\varepsilon \in] 0,1], 2 \mathrm{e}^{1 / 4} \leqslant 3$ and for $n \varepsilon \leqslant 2$, we have that $3 \mathrm{e}^{-n \varepsilon^{2} / 8} \geqslant 1$. The law of large numbers now comes from Borel-Cantelli's lemma. 
To prove the central limit theorem and the law of the iterated logarithm, we will need a weaker version of the law of large numbers (to show that the variance converges). This will be the following

Lemma 4.9. Let $\rho$ be an absolutely continuous probability measure on $\mathbb{R}$ having an moment of order $2+\varepsilon$ for some $\varepsilon \in \mathbb{R}_{+}^{*}$ and such that $0<\int_{\mathbb{R}_{+}} y \mathrm{~d} \rho(y)<\int_{\mathbb{R}_{-}}(-y) \mathrm{d} \rho(y)$.

For any borelian and bounded function $f$ on $\mathbb{R}_{+}$and any $x \in \mathbb{R}_{+}$,

$$
\frac{1}{n} \sum_{k=0}^{n-1} f\left(X_{k}\right) \rightarrow \int f \mathrm{~d} \nu \mathbb{P}_{x}-\text { a.e. }
$$

Remark 4.10. In this lemma, we don't ask the function $f$ to be directly Riemann-integrable but the price we have to pay is a stronger moment hypothesis on $\rho$.

Proof. Write $f=g-P g+\int f \mathrm{~d} \nu$ with $g$ the function given by Proposition 4.6 and such that

$$
C(g)=\sup _{x} \frac{|g(x)|}{1+|x|}<+\infty
$$

Then,

$$
\frac{1}{n} \sum_{k=0}^{n-1} f\left(X_{k}\right)=\int f \mathrm{~d} \nu+\frac{1}{n}\left(g\left(X_{0}\right)-g\left(X_{n}\right)\right)+\frac{1}{n} \sum_{k=0}^{n-1} g\left(X_{k+1}\right)-P g\left(X_{k}\right) .
$$

Moreover, for any $s \in[1,+\infty[$,

$$
\mathbb{E}_{x}\left|g\left(X_{k+1}\right)-P g\left(X_{k}\right)\right|^{s} \leqslant 2^{s} P^{k+1}|g|^{s}(x) \leqslant 2^{s} C(g)^{s} P^{k+1} u_{s}(x)
$$

with $u_{s}(x)=(1+x)^{s}$.

But, doing the same computations as in the proof of Lemma 4.6, we see that if $\rho$ has a moment of order $s+1$, then there are $C, B \in \mathbb{R}_{+}^{*}$ such that

$$
u_{s} \leqslant C\left(u_{s+1}-P u_{s+1}+B\right)
$$

so,

$$
\begin{aligned}
& \sum_{n=1}^{+\infty} \frac{1}{n^{s}} \mathbb{E}_{x}\left|g\left(X_{k+1}\right)-P g\left(X_{k}\right)\right|^{s} \leqslant C C(g)^{s} 2^{s} \sum_{n=1}^{+\infty} \frac{1}{n^{s}} P^{n+1}\left(u_{s+1}-P u_{s+1}+B\right) \\
& \leqslant C 2^{s} C(g)^{s}\left(\sum_{n=1}^{+\infty} \frac{P^{n+1}\left(I_{d}-P\right) u_{s+1}}{n^{s}}+B \sum_{n=1}^{+\infty} \frac{1}{n^{s}}\right) \leqslant C 2^{s} C(g)^{s}\left(u_{s+1}+B \sum_{n=1}^{+\infty} \frac{1}{n^{s}}\right)<+\infty
\end{aligned}
$$

and this proves, using the law of large numbers for martingales (see Thm. 2.18 in [4]) that if $\rho$ has a moment of order $2+\varepsilon$ for some $\varepsilon \in \mathbb{R}_{+}^{*}$, then for any $x \in \mathbb{R}$,

$$
\frac{1}{n} \sum_{k=0}^{n-1} g\left(X_{k+1}\right)-P g\left(X_{k}\right) \rightarrow 0 \mathbb{P}_{x} \text {-a.e. and in } \mathrm{L}^{1}\left(\mathbb{P}_{x}\right) .
$$

Doing the same kind of computations, we also prove that $\frac{1}{n} g\left(X_{n}\right)$ converges to 0 and this proves the expected result.

Theorem 4.11. Let $\rho$ be an absolutely continuous probability measure on $\mathbb{R}$ having an moment of order $2+\varepsilon$ for some $\varepsilon \in \mathbb{R}_{+}^{*}$ and such that $0<\int_{\mathbb{R}_{+}} y \mathrm{~d} \rho(y)<\int_{\mathbb{R}_{-}}(-y) \mathrm{d} \rho(y)$. 
For any directly Riemann-integrable function $f$ on $\mathbb{R}_{+}$with $\int f \mathrm{~d} \nu=0$ we denote $g$ the bounded function given by Corollary 4.7 and such that $f=g-P g$ and we set

$$
\sigma^{2}=\int_{\mathbb{R}_{+}} g^{2}-(P g)^{2} \mathrm{~d} \nu
$$

and for any $x \in \mathbb{R}_{+}$and any $n \in \mathbb{N}^{*}$, we set

$$
S_{n} f(x)=\sum_{k=0}^{n-1} f\left(X_{k}\right)
$$

where $\left(X_{n}\right)$ is the reflected random walk defined by $\rho$ and starting at $x$.

Then, if $\sigma^{2}=0$, we have that for a.e. any $x \in \mathbb{R}_{+},\left(S_{n} f(x)\right)$ is bounded in $\mathrm{L}^{\infty}\left(\mathbb{P}_{x}\right)$.

Moreover, if $\sigma^{2} \neq 0$, we set, for $t \in[0,1]$ and any $x \in \mathbb{R}_{+}$,

$$
\xi_{n}(t)=\frac{1}{\sqrt{n}}\left(S_{i} f(x)+n\left(t-\frac{i}{n}\right) f\left(X_{i}\right)\right) \quad \text { for } \quad \frac{i}{n} \leqslant t \leqslant \frac{i+1}{n} \quad \text { and } \quad 0 \leqslant i \leqslant n-1
$$

and we have that, for any bounded continuous function $F: \mathcal{C}^{0}([0,1]) \rightarrow \mathbb{R}$,

$$
\mathbb{E} F\left(\xi_{n}\right) \rightarrow \mathbb{E} F\left(W_{\sigma^{2}}\right) \text { and } \frac{1}{\ln n} \sum_{k=1}^{n} \frac{1}{k} F\left(\xi_{k}\right) \rightarrow \mathbb{E} F\left(W_{\sigma^{2}}\right) \text { a.e. }
$$

Where we denoted $W_{\sigma^{2}}$ the Wiener measure with variance $\sigma^{2}$.

Moreover, for any a.e. continuous function $\varphi$ on $\mathbb{R}$ such that $x^{2} \varphi(x)$ is bounded,

$$
\frac{1}{\ln n} \sum_{k=1}^{n} \frac{1}{k} \varphi\left(\frac{S_{k} f(x)}{\sqrt{k}}\right) \rightarrow \mathbb{E} \varphi\left(W_{\sigma^{2}}(1)\right) \text { a.e. }
$$

Proof. First, we use that

$$
\sum_{k=0}^{n-1} f\left(X_{k}\right)=g\left(X_{0}\right)-g\left(X_{n}\right)+\sum_{k=0}^{n-1} g\left(X_{k+1}\right)-P g\left(X_{k}\right)
$$

Let $M_{n}=\sum_{k=0}^{n-1} g\left(X_{k+1}\right)-P g\left(X_{k}\right)$. Then, $M_{n}$ is a martingale with bounded difference sequence.

Moreover, noting $\left(\mathcal{F}_{n}\right)$ the filtration associated to this martingale, we have that

$$
\begin{aligned}
\mathbb{E}_{x}\left[\left(M_{n+1}-M_{n}\right)^{2} \mid \mathcal{F}_{n}\right] & =\mathbb{E}_{x}\left[g\left(X_{n+1}\right)^{2}-g\left(X_{n+1}\right) P g\left(X_{n}\right)+\left(P g\left(X_{n}\right)\right)^{2} \mid \mathcal{F}_{n}\right] \\
& =P\left(g^{2}\right)\left(X_{n}\right)-(P g)^{2}\left(X_{n}\right)
\end{aligned}
$$

And so,

$$
\frac{1}{n} \sum_{k=0}^{n-1} \mathbb{E}_{x}\left[\left(M_{k+1}-M_{k}\right)^{2} \mid \mathcal{F}_{k}\right]=\frac{1}{n} \sum_{k=0}^{n-1} P g^{2}\left(X_{k}\right)+\frac{1}{n} \sum_{k=0}^{n-1}(P g)^{2}\left(X_{k}\right)
$$

But, the function $g$ is bounded on $\mathbb{R}$, so, according to Lemma 4.9 ,

$$
\lim _{n \rightarrow+\infty} \frac{1}{n} \sum_{k=0}^{n-1} \mathbb{E}_{x}\left[\left(M_{k+1}-M_{k}\right)^{2} \mid \mathcal{F}_{k}\right]=\int g^{2} \mathrm{~d} \nu-\int(P g)^{2} \mathrm{~d} \nu \mathbb{P}_{x}-\text { a.e. and in } \mathrm{L}^{1}\left(\mathbb{P}_{x}\right)
$$


Moreover, we can compute

$$
\begin{aligned}
& \int_{\mathbb{R}} \int_{\mathbb{R}}(P g(x)-g(|x+y|))^{2} \mathrm{~d} \nu(x) \mathrm{d} \rho(y) \\
& \quad=\int_{\mathbb{R}}(P g(x))^{2} \mathrm{~d} \nu(x)-2 \int_{\mathbb{R}} \int_{\mathbb{R}} P g(x) g(|x+y|) \mathrm{d} \nu(x) \mathrm{d} \rho(y)+\int_{\mathbb{R}} \int_{\mathbb{R}} g^{2}(|x+y|) \mathrm{d} \nu(x) \mathrm{d} \rho(y) \\
& \quad=\int_{\mathbb{R}} g^{2} \mathrm{~d} \nu-\int_{\mathbb{R}}(P g)^{2} \mathrm{~d} \nu=\sigma^{2}
\end{aligned}
$$

So, if $\sigma^{2}=0$, then for $\nu$-a.e. $x \in \mathbb{R}_{+}$and $\rho$-a.e. $y \in \mathbb{R}, P g(x)=g(|x+y|)$. In particular, for a.e. $x \in \mathbb{R}$, $g\left(X_{k+1}\right)=P g\left(X_{k}\right)$ a.e. and this proves that $M_{n}=0$ a.e. Thus, in this case, for a.e. $x \in \mathbb{R}_{+}, S_{n} f(x)=$ $g(x)-g\left(X_{n}\right)$ a.e. and this proves that $\left(S_{n} f(x)\right)$ is bounded in $\mathrm{L}^{\infty}\left(\mathbb{P}_{x}\right)$ since $g$ is bounded on $\mathbb{R}_{+}$.

Finally, is $\sigma^{2} \neq 0$, using the functional central limit theorem (see Thm. 4.1 in part 4.2 of [4]) and the almost sure functional central limit theorem (see [1], Thm. 2.1 and 2.4) we get the expected result since $g$ is bounded and so, $g\left(X_{n}\right) / \sqrt{n}$ converges to 0 a.e. and in $\mathrm{L}^{1}\left(\mathbb{P}_{x}\right)$.

\section{REFERENCES}

[1] F. Chaabane, Version forte du théorème de la limite centrale fonctionnel pour les martingales. C. R. Acad. Sci. Paris Sér. I Math. 323 (1996) 195-198.

[2] W. Feller, An introduction to probability theory and its applications. Vol. II. 2nd edition. John Wiley and Sons, Inc., New York London Sydney (1971).

[3] P.W. Glynn and S.P. Meyn, A Liapounov bound for solutions of the Poisson equation. Ann. Probab. 24 (1996) $916-931$.

[4] P. Hall and C.C. Heyde, Martingale limit theory and its application. Probability and Mathematical Statistics. Academic Press Inc. [Harcourt Brace Jovanovich Publishers], New York (1980).

[5] U. Krengel, Ergodic theorems. With a supplement by Antoine Brunel. Vol. 6 of de Gruyter Studies in Mathematics. Walter de Gruyter and Co., Berlin (1985).

[6] J.P. Leguesdron, Marche aléatoire sur le semi-groupe des contractions de $\mathbf{R}^{d}$. Cas de la marche aléatoire sur $\mathbf{R}_{+}$avec choc élastique en zéro. Ann. Inst. Henri Poincaré Probab. Statist. 25 (1989) 483-502.

[7] S.P. Meyn and R.L. Tweedie, Markov chains and stochastic stability. Communications and Control Engineering Series. Springer Verlag London Ltd., London (1993).

[8] M. Peigné and W. Woess, On recurrence of reflected random walk on the half-line. With an appendix on results of Martin Benda. Preprint arXiv:math/0612306 (2006).

[9] F. Spitzer, Principles of random walk. The University Series in Higher Mathematics. D. Van Nostrand Co., Inc., Princeton, N.J. Toronto London (1964). 